250 Internat. Konf. f. Krebsforschung, Sept. 1906 zu Heidelberg u. Frankfurt a. $M$.

Vieles schon getan worden. Das Geschehene genügt aber bei weitem noch nicht, und es muss in dieser Hinsicht noch viel mehr unternommen werden, dem es unterliegt keinem Zweifel, dass auf diese Weise die Zahl der durch frühzeitige und richtige Behandlung heilbaren Krebskranken sehr beträchtlich erhöht werden kann. Auch betreff's der Pflege und Fürsorge der unheilbaren gilt es ja als allgemein anerkannt, dass wir erst an Anfange umserer Tätigkeit stehen.

So möchte ich es mir denn erlauben, meine Herren, Sie dringend zum Kampfe aufzurufen und darauf hinzuweisen, dass thmen allen fast täglich, je nach ihrem spezialfache, in den verschiedensten Richtungen hin Gelegenheit geboten wird, sei es durch Tat oder Wort, mächtig und wirkungsvoll einzugreifen. Nur wenn allgemein die Erkenntnis herrscht, dass die Krebsbekämpfung zurecht besteht und dass der Krebs durch Frühund Radikalbehandlung heilbar ist, werden wir unserem Ziele wirklich näher getreten sein. Bis dahin aber müssen wir alle rastlos weiter wirken und uns nicht durch den falschen Gedanken zu ablehnender Passivität rerleiten lassen. dass wir in der Wüste predigen, denn mit Geduld und Glauben wïchst und entwickelt sich die geringste Uase zum herrlichsten Wald. Bleiben unsere Bemühungen nur zu oft noch erfolglos; so genügt doch der kleinste hier über den Feind errungene Sieg, um sie mehr als zu rechtfertigen, und erscheint uns das lange Kämpfen wohl manchmal als ein undankbares Lnternehmen, so dürfen wir uns ja doch schliesslich, heute mehr denn je, mit der Hoffinung trösten, der Tag werde wohl kommen, an welchem wir dem Krebse mit anderen, besseren Waffen entgegentreten können!

br. A. Béclère, Médecin de l'hèpital Saint-Antoine (Paris):

\title{
La radiothérapie des tumeurs malignes sous-cutanées; les conditions du succès et de l'insuccès de cette médication.
}

l)ans la lutte contre le cancer; lemploi thérapeutique des rayons de Rüntgen constitue un précieux auxiliaire de lintervention chirmgicale.

Personne ne peut plus contester laction curative des ces rayons sur la grande majorité des épithéliomes de la peau.

Par contre beaucoup des médecins ne croient pas encore que ces rayons puissent, au travers de la peau saine, agir favorablement sur des tumeurs malignes sous-cutanées.

Cependant des faits bien observes démontrent que les rayons de Röntgen sont capables de faire disparaitre certaines-tumeurs épithélionateuses de la glande mammaire, certaines tumeurs épithéliomateuses des ganglions Jymphatiques de laisselle ou du cou, certaines tumeurs sarco- 
mateuses des diverses régions, toutes productions néoplasiques ayant le caractire commun dètre développées an dessous du tégument cutané et dêtre recomvertes par une peau non ulcérée.

Il faut recomaitre que de tels faits ne sont pas encore trés nombreux, que dans la foule des cas analogues, traités sans succès par la radinthérapie, ils constituent plutiot une exception. Cependant ils existent, on ne saurait les nier. II importe donc de passer en revue les conditions multiples, dordre tres différent, dont dépendent, dans la radiothérapie des tumeurs malignes sous-cutanées, le succès ou l'insuccés de cette médication.

La radiothérapie des néoplasmes n'est qu'une partie de la radiothérapie en général; celle-ci elle-même n'est qu'un chapitre de ce quion peut appeler la hio-radiologie, cest a dire de notre science des actions produites sur l'ensemble des itres vivants et, plus particulierement sur Thomme, sain ou malade, d'une part par les rayons de Röntgen, d'autre part par les rayons du radium et des autres substances radio-actives.

l.a radiothérapie des néoplasmes est donc soumise aux lois générales: les unes dordre pmement physique, les autres dordre biologique, qui régissent laction des radiations nouvelles sur les éléments cellulaires de lorganisme.

Ces lois fondamentales sont au nombre de trois:

1. Tout élément cellulaire vivant, sain on malade, s̈il absorbe une 'juantité suffisante de rayons de Röntgen, devient le siège de transformations chimiques dont le dernier terme est la dégénérescence et la mort de lélément irradié;

2. Les divers élements cellulaires vivants, sains ou malades, sont trés inégalement sensibles a l'action des rayons de Röntgen; en d'autres termes; la quantiti des rayons de Röntgen dont l'absorption est nécessaire pour amener la dégénérescence et la mort des divers éléments cellulaires se montre très différente suivant qu’il s'agit d'éléments épithéliaux, musculaires, cartilagineux, des globules rouges, des leucocytes, etc.

3. Lá quantité des rayons de Röntgen successivement absorbée par les direrses couches dune même région irradiée ra toujours en décroissant tres rapidement de la superficie vers la profonden. Pour préciser, cest tout ou plus, dans les conditions les plus favorables, si la quantité absorbée :a un centimetre de profondeur au dessous de la conche épidermique superficielle, dépasse la moitié de la quantité absorbée par cette dernière.

On peut dire de cette derniere loi, dordre purement physique, que ¿est lobstacle, jusqua présent an moins, insurmontable qui soppose aux progrès de la radiothérapie.

Par contre et fort heureusement, les éléments cellulaires dont sont composés les néoplasmes se montrent, dune manière générale, notablement 
252 Internat. Konf. f. Krebsforschung, Sept. 1906 zu Heidelberg u. Frankfurt a. M.

plus sensibles a laction destructive des rayons de Röntgen que les éléments sains qui les entourent.

C'est pour cette raison que les rayons de Röntgen peuvent, comme les caustiques, détruire, par exemple. tous les éléments cellulaires diun cancer de la peau. mais ils diffèrent des caustiques en ce qüils ne détruisent par les tissus en masse, ils detruisent les cellules malades tout en respectant les cellules saines aroisinantes, moins sensibles, en un mot leur action est une action élective.

Cest pour cette raison aussi que les rayons de Röntgen peurent. comme le fait est bien démontré, détruire, cellule par cellule. certains néoplasmes sous-cutanés, par exemple certains épithéliomes de la glande mammaire ou certains sarcomes, sans détruire cependant la peaa saine qui les recourre, manifestant ainsi ou plus haut degré cette action élective dont les caustiques sont nécessairement dépourrus.

En principe, tont néoplasme sous-cutané devrait pouvoir être détruit par la radiothérapie, il suffirait qu'il absorbat, dans ce but, une quantité de rayons de Röntgen suffisante.

En fait, il en ra tout autrement, un petit nombre senlement des néoplasmes sous-cutanés est traité avec avantage par Ia radiothérapie et si on étudie les conditions multiples et dordre très different dont dépendent. dans chaque cas particulier, le succes ou l“insuccés de cette médication. on peut les réduire à trois principales.

Ia première est d'ordre purement physique, c'est le siege plus ou moins profonds des divers éléments cellulaires du néoplasme primitif au dessous du revètement cutaué ou muqueux.

La seconde, dordre biologique et pathologique, cest l'évolution plus on moins rapide des foyers néoplasiques ou, en d'antres termes, la multiplication plus ou moins rapide des éléments cellulaires dont ils sont formés.

La troisieme enfin. d'ordre biologique et anatomique, cest l'intégrité ou l'envahissement des vaisseaux lymphatiques de la région malade et des ganglions, plus ou moins profondement situés, auxquels aboutissent ces vaisseaux.

Le siège en profondeur du néoplasme. Le ròle de la première condition est, on le comprend facilement, des plus importants. Puisque les quantités des rayons de Röntgen, successivement absorbées par les diverses couches des tissus traversés, vont en décroissant très rapidement de la superficie vers la profondeur. un foyer néoplasique cesse nécessairement, a une certaine profondeur au dessous de la surface épidermique, d'être accessible à la radiothérapie, parce que le médecin, désireux de le traiter, se trouve enfermé dans ce dilemme: ou bien donner au foyer morbide la dose curative sans se soucier de léser gravement la peau saine 
¿ui le recourre ou bien veiller à la conservation de la peau sans atteindre la dose nécessaire it la guérison des lésions sous-jacentes.

Pratiquement, c'est toujours a ce demier parti quil doit se ranger.

La profondeur à laquelle un néoplasme cesse d'être accessible au traitement varie d'ailleurs avec son degré de sensibilité aux rayons de Röntgen. Pour tel nodule épithéliomateux cette profondeur critique commence immédiatement au dessous du derme tandisque pour tel volumineux sarcome, d'une sensibilité beaucoup plus grande, elle n'est pas dépassée même dans le médiastin.

Lne technique. correcte ou defectueuse, etend aussi plus ou moins en profondeur le champ de l'action thérapeutique. Pour en atteindre l'extrême limite, trois moyens sont concurremment employés: la position de l'ampoule de Röntgen à grande distance de la surface traitée, le choix diune ampoule très-résistante donnant des rayons trés-pénétrants, la filtration des rayons ¿l'aide d'une mince feuille d'almminium placée entre leur foyer d'émission et la peau. On a soin igalement, dans tous les cas où cette tactique est possible, dattaquer le néoplasme par plusieurs roies, de le prendre, pour ainsi dire, entre plusieurs feux convergents. Ainsi, pour le traitement dun néoplasme du médiastin a l'exemple du docteur Kienböck, de Vienne, on subdivise toute la surface cutanée du thorax y compris les régions susclaviculaire et axillaire, en In certain nombre de circonscriptions dont chacune sert tour à tour de porte d'entrée aux rayons, tandisque les autres sont protégées par une feuille de plomb; cest le moyen de multiplier la dose donnée aux néoplasmes profonds sans dépasser pour l'enveloppe tégumentaire la dose tolérable.

En dépit de ces moyens, la rapide décroissance des doses absorbées, de la superficie vers la profondeur, nen constitue pas moins la loi physique inexorible ‘̀ laquelle viennent se heurter jusqu’à présent, comme à un mur dairain, les efforts des radiothérapeutes. L'introduction dans les tissus irradiés de solutions, telles que les solutions de séls de quinine, capables de devenir fluorescentes sous l'action des rayons de Röntgen n'a pas réussi, comme on l'esperait, a renforcer sîrement leur action thérapeutique.

D'aprés cette loi fatale, certains néoplasmes ne recoivent jamais, en tous les points de leur masse, qu une dose inférieure à la dose curative, aussi leur évolution n'est-elle pas entravée par le traitement.

D'autres recoivent bien la dose curative, mois, en raison de leur epaisseur, ils la recoivent seulement dans les couches les plus roisines de la peau: ils regressent donc a la surface mois continuent à progresser dans la profondeur. Pour la même raison, alors que la masse principale diun néoplasme parait devoir completement disparaitre sous l'influence du traitement, il suffit parfois d'un minime prolongement, un peu plus profond, 
254 Internat. Konf. f. Krebsforschung, Sept. 1906 zu Heidelberg u. Frankfurt a. H.

pour tout remettre en question et pour compromettre irrémediablement une guérison qui semblait prochaine.

La rapidité dévolution du néoplasme. Céefacteur, dordre biologique, la plus ou moins grande rapidité d’écolution du néoplasme ou. plus exactement, le degré de rapidité avec laquelle se multiplient les eléments cellulaires dont il est formé, ne joue pas un role moins important que le facteur physique de la profondeur al laquelle s'étend le néoplasme. En effet, si la première irradiation ne suffit pas à tuer toutes les cellules malades mais ne les altere que faiblement, on comprend que les résultats du traitement seront très différents suivant que daus lintervalle entre deux séances consécutives, les cellules primitivement irradiées se seront multipliées ou quil ne sera survenu aucane production de cellules nouvelles. Dans le premier cas, il est impossible despérer la guérison: dans le second cas, au contraire, l'absorption prolongè de faibles doses par des cellules qui ne prolifèrent pas peut aboutir, avec l'aide du temps, ¿ leur complète destruction. La radiothérapie est toujours une médication Jente, a longue échéance; rien ne sert mieux son action quane lente évolution de la maladie, rien n'y met plus obstacle que la marche rapide de cancer.

I. envahissement du système lymphatique. - Enfin il sert pen que, sous linfluence des rayons, la tumeur primitive récresse entierement si déjà le systeme lymphatique esi envahi, si déjà le transport et la grefte des éléments néoplasiques ont créé des foyers secondaires dans les ganglions les plus proches ou si, en raison de la multiplication rapide des éléments neoplasiques, ces foyers apparaissent au cours du traitement.

Quadvient-il alors: Tout dépend des dispositions anatomiques de la région. Si les ganglions secondairement atteints nont pas un siegge plus profond que la tumeur primitive, ils peuvent, comme celle-ci, ceder a la radiothérapie. Ainsi chez les malades, atteintes de cancel du sein, on réussit, en certains cas, ¿̀ faire disparaitre les ganglions secondaires de même nature superficiellement situés sous lá peau de liaisselle on sous la peau de la base du cou, tandisqu'on agit plus rarement sur les ganglious moins superficiels qui accompagnent les vaisseaux sons-claviers et que. presque sans exception, on demeure désarmé vis-it-vis des ganglions profonds du médiastin.

Dans le cancer du sein, mème apres les premieres etapes de l'enrahissement lymphatique, en raison du siege relativement superficiel des ganglions atteints, la radiothérapie peut encore, en certains cas at marche lente, se montrer efficace. Tout au contraire, pour un organe tel que le col utérin, plus directement accessible au traitement que la glande nammaire, mais dont les vaisseaux lymphatiques aboutissent i des ganglions: profondément situés et par suite inaccessibles, la radiothérapie arrive trop 
tard dès que la lésion a franchi le derme de la muqueuse. comme ciest malheureusement presque toujours le cas au moment où est porté le diagnostic.

Le diagnostic précoce. - Dans ces conditions, on comprend combien il importe à l'efficacité du traitement qu'il soit institué de bonne heure, combien par conséquent un diagnostic précoce est nécessaire et combien pour certains organes, pour certains régions cette nécessité s'impose plus étroitement que pour dautres.

Ainsi, comme nous venons de le dire, il nest pas encore tout i fait trop tard pour espérer les bienfaits du traitement dans un cas de cancer fu sein, quand déjà les ganglions superficiels au dessus de la clavicule sont atteints pourvu toutefois que cette étape ganglionnaire, la troisième ou la seconde ou moins sur le trajet de l'envahissement des lymphatiques, ne soit pas dépassée.

Pat contre, on pent imaginer une tumeur de lestomac qui soit en apparence, au point de vue de la radiothérapie, dans des conditions ideales, $\therefore$ est if dire mne tumeur de tres petit volume, provenant de la face anterieure de l'organe, en rapport direct avec la paroi abdominale, sans interposition du foie, recouverte seulement, chez un sujet maigre et peu muscle par une très faible épaisseur des parties molles. Même dans ces conditions dont la réunion est à préroir si exceptionelle, on peut prédire presque it coup sir que les ganglions profonds, inaccessibles an traitement, seront déja envahis au moment ou celui-ci débutera et que, dans le cas où il produirait une amélioration temporaire, comme cela a été observé, dans le cas mème ou il ferait disparaitre la tumeur primitive, cependant il natteindra pas finalement le but espéré, si précoce quait été le diagnostic.

Au total, la sensibilité plus on moins vive des éléments néoplasiques a laction des rayons de Röntgen, le siegge plus ou moins profond de ces éléments au dessous de la surface tégumentaire, leur plus ou moins grande rapidité de multiplication, la localisation du mal au foyer primitif, sans participation du système lymphatique ou lenvahissement à distance de ganglions tantit accessibles et tantôt inaccessibles au traitement, sans parler de la correction plus ou moins parfaite de la technique et du dosage employés, tels sont les facteurs principaux dont dépendent, dans la radiothérapie des tumeurs malignes sous-cutanées, le succès ou linsuccès final.

Sans entrer dans une étude détaillée des tumeurs malignes souscutanées, ćest le cancer du sein, à toutes les périodes de son évolution et ce sont les sarcomes qui pourront surtout bénéficier de la radiothérapie, dans une plus ou moins large mesure et pour une durée plus on moins longne.

11 est démontré que dans certaines conditions dont la principale est la lenteur d'érolution de la maladie, un cancer du sein peut disparaitre 
256 Internat. Konf. f. Krebsforschung, Sept. 1906 zu Heidelberg u. Frankfurt a. M.

sans opération, sous linfluence de la radiotbérapie seule, quand il est encore limité a la glande mammaire on même, par exception, yluand les ganglions superficiels sont déjà envahis.

Après l'intervention chirurgicale, la radiothérapie se montre très efficace, dans le cancer du sein, non seulement sur les récidives cutanées sous forme de nodosités et d'ulcérations qu'elle fait disparaitre, mais aussi sur les nodosités sous-cutanées de récidive et même, dans certains cas, sur les ganglions secondaires les plus superficiels; elle demeure malheureusement impuissante contre l'envahissement profond du systeme lymphatique, de la cage thoracique et de son contenu.

Dans tous les cas de cancers du sein jugés inopérables demblee ou après une première intervention suivie de récidive, la radiothẻrapie demeure le traitement de choix, capable de donner une guérison locale, daméliorer l'état général et de prolonger la vie, tout ou moins d'en alléger les souffrances et d'en soutenir les dernières illusions.

La radiothérapie exerce aussi une action favorable et sourent curative sur un assez grand nombre de sarcomes sous-cutanes; ils se montrent, d'une manière générale, plus sensibles à son action que les épithéliomes et même certains d'entre eux se distinguent par une sensibilité tout a fait extruordinaire à l'égard des rayons de Röntgen. Cette extrème sensibilité, la marche souvent lente de ces néoplasmes, labsence ou la date tardive de l'envabissement du système lymphatique, telles sont les raisons iprincipales qui permettent de comprendre les cas encore rares mais incontestables de guerison obtenus par la radiothérapie dans certains sarcomes volumineux et profonds.

En résumé. Dans le domaine du traitement des tumeurs, malignes sous-cutanées, le champ de la radiothérapie est incontestablement limité par la série des conditions qui ont été étudiées plus haut. Si limité qu'il soit, il ne doit pas cependant etre négligé et mérite dattirer toute lattention du chirurgien. La radiothérapie est rarement capable de suppléer à son intervention, mais elle peut souvent compléter son oeuvre avec grand avantage et en prolonger l'action bienfaisante.

C'est pourquoi son Excellence II. le Professeur Czerny roudra bien me permettre de le féliciter de la place qu'il a donnée à la radiothérapie dans son nouvel et admirable Institut pour le traitement du cancer. C'est ¿ l'union de la chirurgie et de la radiothérapie que nous devrons, je crois, les plus beaux succés thérapeutiques en attendant le jour dont il est permis d'espérer la venue oi les travaux de M. le Professeur Leyden, et les recherches expérimentales, si pleines de promesses, de $M$. le Professeur Ehrlich sur limmunisation anti-cancé:euse auront porté tous leur fruits, le jour rêvé oil le bistouri et les rayons de Röntgen seront devenus contre le cancer, des armes superflues. 